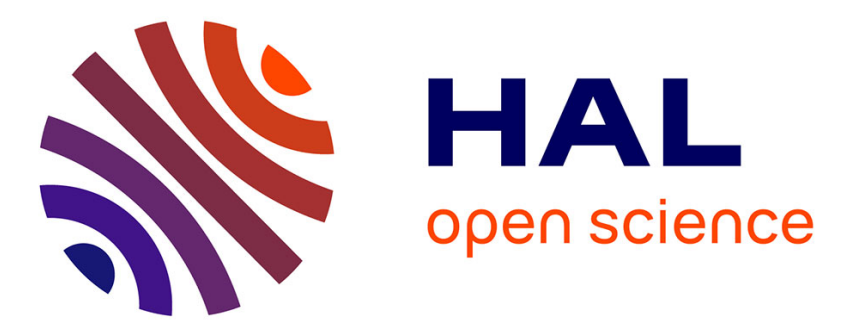

\title{
Ion dynamics in compacted clays: Derivation of a two-state diffusion-reaction scheme from the lattice Fokker-Planck equation
}

Benjamin Rotenberg, Jean-François Dufrêche, B. Bagchi, E. Giffaut, J.-P Hansen, P. Turq

\section{To cite this version:}

Benjamin Rotenberg, Jean-François Dufrêche, B. Bagchi, E. Giffaut, J.-P Hansen, et al.. Ion dynamics in compacted clays: Derivation of a two-state diffusion-reaction scheme from the lattice FokkerPlanck equation. Journal of Chemical Physics, 2006, 124 (15), pp.154701. 10.1063/1.2194014 . hal01897033

\section{HAL Id: hal-01897033 \\ https://hal.sorbonne-universite.fr/hal-01897033}

Submitted on 16 Oct 2018

HAL is a multi-disciplinary open access archive for the deposit and dissemination of scientific research documents, whether they are published or not. The documents may come from teaching and research institutions in France or abroad, or from public or private research centers.
L'archive ouverte pluridisciplinaire HAL, est destinée au dépôt et à la diffusion de documents scientifiques de niveau recherche, publiés ou non, émanant des établissements d'enseignement et de recherche français ou étrangers, des laboratoires publics ou privés. 
Ion dynamics in compacted clays: Derivation of a two-state diffusion-reaction scheme from the lattice Fokker-Planck equation

B. Rotenberg, J.-F. Dufrêche, B. Bagchi, E. Giffaut, J.-P. Hansen, and P. Turq

Citation: The Journal of Chemical Physics 124, 154701 (2006); doi: 10.1063/1.2194014

View online: https://doi.org/10.1063/1.2194014

View Table of Contents: http://aip.scitation.org/toc/jcp/124/15

Published by the American Institute of Physics

\section{Articles you may be interested in}

Solvation of complex surfaces via molecular density functional theory

The Journal of Chemical Physics 137, 224107 (2012); 10.1063/1.4769729

Structural properties of water: Comparison of the SPC, SPCE, TIP4P, and TIP5P models of water

The Journal of Chemical Physics 123, 104501 (2005); 10.1063/1.2018637

Dynamical effects in electron transfer reactions

The Journal of Chemical Physics 84, 4894 (1986); 10.1063/1.449978

Role of quantum coherence in shaping the line shape of an exciton interacting with a spatially and temporally correlated bath

The Journal of Chemical Physics 146, 194902 (2017); 10.1063/1.4983223

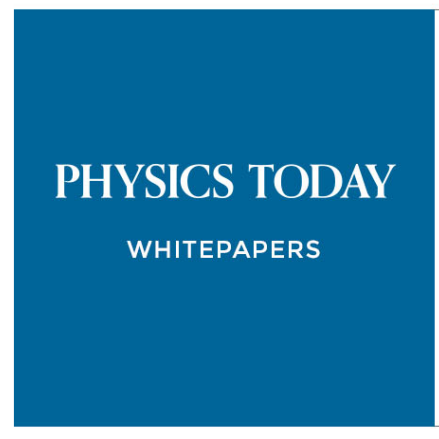




\title{
Ion dynamics in compacted clays: Derivation of a two-state diffusion- reaction scheme from the lattice Fokker-Planck equation
}

\author{
B. Rotenberg ${ }^{\text {a) }}$ \\ UMR 7612, Laboratoire LI2C, Université Pierre et Marie Curie-Paris 6, Case Courrier 51, \\ 4 Place Jussieu, Paris F-75005, France and ANDRA, Parc de la Croix Blanche, 1/7 Rue Jean Monnet, \\ 92298 Châtenay Malabry Cedex, Paris F-75005, France \\ J.-F. Dufrêche \\ UMR 7612, Laboratoire LI2C, Université Pierre et Marie Curie-Paris 6, Case Courrier 51, \\ 4 Place Jussieu, Paris F-75005, France \\ B. Bagchi \\ Solid State and Structural Chemistry Unit, Indian Institute of Science, Bangalore 560012, India \\ E. Giffaut \\ ANDRA, Parc de la Croix Blanche, 1/7 Rue Jean Monnet, 92298 Châtenay Malabry Cedex, \\ Paris F-75005, France \\ J.-P. Hansen \\ Department of Chemistry, University of Cambridge, Lensfield Road, Cambridge CB2 1EW, \\ United Kingdom \\ P. Turq \\ UMR 7612, Laboratoire LI2C, Université Pierre et Marie Curie-Paris 6, Case Courrier 51, \\ 4 Place Jussieu, Paris F-75005, France
}

(Received 6 January 2006; accepted 17 March 2006; published online 17 April 2006)

\begin{abstract}
We show how a two-state diffusion-reaction description of the mobility of ions confined within compacted clays can be constructed from the microscopic dynamics of ions in an external field. The diffusion-reaction picture provides the usual interpretation of the reduced ionic mobility in clays, but the required partitioning coefficient $K_{d}$ between trapped and mobile ions is generally an empirical parameter. We demonstrate that it is possible to obtain $K_{d}$ from the microscopic dynamics of ions interacting with the clay surfaces by evaluating the ionic mobility using a novel lattice implementation of the Fokker-Planck equation. The resulting $K_{d}$ allows a clear-cut characterization of the trapping sites on the clay surfaces and determines the adsorption/desorption rates. The results highlight the limitations of standard approximation schemes and pinpoint the crossover from jump to Brownian diffusion regimes. (C) 2006 American Institute of Physics. [DOI: 10.1063/1.2194014]
\end{abstract}

\section{INTRODUCTION}

Ion mobility near surfaces, and in particular in clays, is generally understood as the result of an interplay between the diffusion of mobile ions and their temporary trapping by surface adsorption sites. The interactions between the ions and the mineral surfaces are the origin of the retention properties of these materials: the vast majority of ions diffuses more slowly in a charged confining medium than in a bulk solution. The reduction of ion mobility in clay materials, in particular montmorillonite, has made them possible candidates for the storage of radioactive ions in nuclear waste. ${ }^{1,2}$ On the macroscopic scale, geologists usually describe the retention properties using the sorption concept: an ion can temporarily adsorb to specific sites on the mineral surfaces, immobilized for some of its time, thus reducing its mobility. This twostate picture, though oversimplified, has the advantage of being easily implemented for engineering applications and is widely used in the extensive literature on the related sorption

\footnotetext{
${ }^{a)}$ Electronic mail: rotenber@ccr.jussieu.fr
}

data (empirical partioning coefficients $K_{d}$ ), thus providing a convenient tool for the modeling and prediction of ion mobility in these materials. ${ }^{1-5}$

From a microscopic point of view there is, however, no clear-cut distinction between bound and mobile species, since the ionic distribution $f(\mathbf{x}, \mathbf{v}, t)$ is a continuous function of position $\mathbf{x}$, velocity $\mathbf{v}$, and time $t$. The purpose of the present investigation is twofold. First, we show how a phenomenological two-state model consistent with the apparent mobility paradigm can be systematically derived from the microscopic interactions between the ions and the clay surfaces. Second, we examine the influence of the strength of a model external interaction potential between the clay surface and the ions on the relevant parameters defining the phenomenological two-state model. These objectives are achieved by describing ion dynamics on a coarse-grained, mesoscopic level based on a recently proposed lattice implementation of the Fokker-Planck equation.,

The paper is organized as follows. Section II summarizes the various levels of modeling ion mobility. Section III introduces the details of the starting, mesoscopic level of de- 
scription and the coarse-graining procedure leading to the diffusion-reaction scheme is described in detail. The lattice Fokker-Planck (LFP) equation is presented in Sec. IV, and its numerical implementation is described for the problem at hand. Its numerical solution determines the apparent mobility of ions confined between two clay layers, which define an external potential surface. The results for the apparent mobility are compared with the predictions of three existing approximations. This apparent mobility is finally used in Sec. $\mathrm{V}$ to construct explicitly the phenomenological two-state model. Concluding remarks are made in Sec. VI.

\section{MOBILE AND ADSORBED IONS IN CLAYS}

\section{A. Diffusion-reaction paradigm}

Ion mobility in clays is usually interpreted as resulting from a balance between the free diffusion of mobile ions and the adsorption of a fraction of available ions at the clay surfaces. The balance between the two species (mobile and trapped) of ions may be schematically represented as a chemical exchange reaction as follows:

$$
\text { mobile } \underset{k_{-}}{\stackrel{k_{+}}{\rightleftarrows}} \text { adsorbed, }
$$

with $k_{+}\left(k_{-}\right)$the adsorption (desorption) rate constant. In a macroscopic tracer diffusion experiment, whose time scale is much longer than the typical adsorption/desorption time, the only relevant parameter is the fraction of adsorbed (mobile) ions, usually described in terms of the distribution coefficient $K_{d}$, defined as the ratio

$$
K_{d}=\frac{[\text { adsorbed }]}{[\text { mobile }]} .
$$

The fraction of adsorbed ions is thus $f_{a}=K_{d} /\left(1+K_{d}\right)$, and that of mobile ions is $f_{m}=1 /\left(1+K_{d}\right)$. The mobility of bound ions is generally assumed to be negligible, and the apparent diffusion coefficient is simply

$$
D_{\text {app }}=f_{a} \times 0+f_{m} \times D_{0}=f_{m} D_{0},
$$

with $D_{0}$ the diffusion coefficient of the mobile ions. The measured (or effective) diffusion coefficient is proportional to $D_{\text {app }}$, but also involves the porosity and the tortuosity, which characterize the morphology of the porous material on large scales. The porosity and tortuosity can be determined from independent measurements, so that we may assume that $D_{\text {app }}$ is experimentally accessible. In fact, (3) can be used in practice to determine the experimental value of $K_{d}{ }^{3-5}$ The other experimental determination of $K_{d}$ consists of "batch" sorption experiments, that is, the titration in solution of nonsorbed ions. ${ }^{2,8,9}$ However, the values obtained in this case are not always consistent with the measured apparent diffusion coefficients, ${ }^{10}$ because of the different environment of the ions in solution and in the confined medium, which is "bulklike" only for high porosities. This discrepancy, if one is not to reject completely the widespread concept of reactive transport to account for the retention properties, pinpoints the need for a better understanding of the meaning of such a two-state picture. The purpose of the present paper is to present how one can extract a value of $K_{d}$ from a microscopic description of ion dynamics in clays.

\section{B. Microscopic description}

On the atomic scale, the distinction between adsorbed and mobile ions is not without its ambiguities. An all-atom description of the motion of ions in the quasi-twodimensional interlayer space of clays can be obtained by molecular dynamics (MD) simulations. ${ }^{11-13}$ This approach has been particularly successful in the determination of the diffusion coefficient of ions $\left(\mathrm{Na}^{+}, \mathrm{Cs}^{+}, \ldots\right)$, and the influence of water content on the latter. More recently, the influence of temperature was also investigated. These studies showed that the diffusion coefficients of ions confined between the clay layers is not very different from those in bulk water $\left(\sim 10^{-9} \mathrm{~m}^{2} \mathrm{~s}^{-1}\right)$ as soon as at least three water layers are formed in the intralamellar space. However, at very low water content (mono- and bilayers), the diffusion coefficients can be reduced by an order of magnitude, and for some ions (e.g., $\mathrm{Cs}^{+}$), the diffusion process is better described by jump diffusion between specific sites on the clay surfaces than by the continuous "liquidlike" behavior of $\mathrm{Na}^{+}$. This effect still persists at elevated temperatures. On the atomic scale, the specific sites can be identified by the presence of three oxygen atoms belonging to the mineral clay layer in the first coordination shell of the $\mathrm{Cs}^{+}$ion. These simulation results (for the diffusion coefficient of water in clays) have been confirmed by quasielastic neutron scattering experiments. ${ }^{14}$

Thanks to thermal motion, induced by the presence of the solvent (and possibly by the phonons in the mineral layer itself), an ion can overcome the energy barrier separating two adjacent adsorption sites. The presence of the mineral layers confines the ions to a quasi-two-dimensional space (the interlayer space), in which these sites are located. Therefore the picture of "bound" and "mobile" ions seems to find its justification at the microscopic level. However, the quantitative definition of the fraction of mobile ions is rather arbitrary, because the ionic density (and the corresponding energy profile) is a continuous function. Furthermore, an arbitrary definition of the sites might not be consistent with the apparent diffusion coefficient defined by (3). Therefore, a relevant definition of the bound and mobile states (and consequently, an operational distribution constant $K_{d}$ ) consists precisely in defining the two states from the value of $K_{d}$; the latter being deduced from the apparent mobility "measured" on the microscopic scale.

\section{COARSE-GRAINING PROCEDURE}

We will describe in detail in Sec. III B the coarsegraining procedure from the microscopic level to the precise definition of the two states, and that of the exchange rate constants. However, we will not start from an atomic description, but rather from an intermediate level of modeling, which we are now going to present.

\section{A. Mesoscopic level}

Using molecular dynamics to directly measure the diffusion coefficient for each temperature, as already done by 
Malikova et al., ${ }^{15,16}$ might not answer the question that we are trying to deal with. The challenge is twofold. The main difficulty is that the very concept of the apparent diffusion coefficient (or equivalently, apparent mobility) needs to compare the observed diffusion coefficient to that of the mobile ions. This raises the issue of the right reference state to compare with. Indeed, in MD simulations the system is defined as a whole and cannot be split into a reference state (mobile ions) and an actual state (mobile and bound ions simultaneously). The second problem arises from the computational demand of such simulations, which make the rigorous study more difficult if one is interested in varying (for example) the temperature over a large range. The reference state issue could be bypassed by simulating a system where the details of interactions of the ions with the mineral layers are switched off. We will not discuss here the technicalities related to such an approach and will just say that we adopted an alternative route in order to simplify the problem. Indeed, a more usual, though approximate, way to study diffusion is to proceed to a first coarse-graining step and adopt a mesoscopic view of the system.

In a mesoscopic description, the solvent is treated as a continuum and the collisions between the solvent molecules and the ions are characterized by a single friction parameter $\gamma$. In addition, the interactions of a particular ion with the surfaces, on the one hand, and with the other ions, on the other hand, are described at the mean-field level by an external potential $V(x, y)$. Finally, the motion of the ions is not described in terms of trajectories (Langevin dynamics), but in terms of a probability density $f(\mathbf{x}, \mathbf{v}, t)$. This mesoscopic treatment simplifies the description of the system by considerably reducing the number of degrees of freedom, and it has proven very efficient to deal with surface diffusion phenomena. ${ }^{17-20}$ The evolution of the probability density is governed by the Fokker-Planck equation ${ }^{21}$ as follows:

$$
\partial_{t} f+\mathbf{v} \cdot \nabla f=\nabla_{\mathbf{v}} \cdot\left(\gamma \mathbf{v}+\frac{\nabla V}{m}+\frac{\gamma k_{B} T}{m} \nabla_{\mathbf{v}}\right) f
$$

with $m$ the mass of the diffusing particle and $k_{B} T$ the thermal energy. This equation treats the ions as independent particles or as interacting particles, though only at the mean-field level, if $V(x, y)$ contains self-consistent interactions. Note that the mean-field self-consistent interaction potential vanishes in the bulk, due to translational invariance, but is nonzero in the presence of interfaces (or space-dependent external potentials), which lead to inhomogeneous density profiles. The evolution of the probability density $f(\mathbf{x}, \mathbf{v}, t)$ gives access to the local ionic density $\rho$ and local velocity $\mathbf{u}$. The density and flux at a given position are indeed the moments of $f$ as follows:

$$
\rho(\mathbf{x}, t)=\int f(\mathbf{x}, \mathbf{v}, t) d \mathbf{v},
$$

$$
\rho(\mathbf{x}, t) \mathbf{u}(\mathbf{x}, t)=\int f(\mathbf{x}, \mathbf{v}, t) \mathbf{v} d \mathbf{v}
$$

The friction is such that in the absence of an external potential and of interactions between ions (e.g., at infinite dilution) the diffusion coefficient of the ions is ${ }^{22}$

$$
D_{0}=\frac{k_{B} T}{m \gamma}=\mu_{0} \frac{k_{B} T}{m} .
$$

The problem of the reference state discussed above is simplified here by the treatment of the interactions as an external potential: the reference state (mobile ions) corresponds to no external potential in the two-dimensional (2D) surface to which the ions are confined. The task is then reduced to the determination of the exact value of the friction from MD simulations. However, we will see that this step is not crucial for the construction of the two-state model, since in the relevant range of friction (high friction regime), the reduced diffusion coefficient $\left(D_{\text {app }} / D_{0}\right)$ or reduced mobility $\left(\mu_{\text {app }} / \mu_{0}\right)$, which is identified with the fraction of mobile ions $f_{m}$, is independent of the value of $\gamma$. This is why we have chosen to solve Eq. (4) for a reasonable choice of $\gamma$ instead of extracting it from simulations. A possible reasonable value would be the Stokes friction, involving the ionic radius and the solvent viscosity. On top of the force $-\nabla V(x, y)$ arising from the interactions with the surfaces, an applied force (e.g., an electric field) can be included in Eq. (4), as will be explained in Sec. III B 1.

In addition to the friction, the second ingredient of the mesoscopic description is the external potential (which could also contain a self-consistent contribution due to the interactions with the other ions). Due to the crystalline structure of clays, the external potential has a hexagonal symmetry. This results in particular in an isotropic diffusion along the clay layers. ${ }^{14}$ If we want to reproduce this feature of the "true" potential, we need to respect this symmetry. The simplest analytical potential with hexagonal symmetry is of the form $^{23}$

$$
V(x, y)=V_{\max } \times v(x, y),
$$

where $v(x, y)$ takes values between 0 and 1 as follows:

$$
\begin{aligned}
v(x, y)= & \frac{1}{3}+\frac{2}{9} \times\left[\cos \frac{2 \pi}{a}\left(x+\frac{y}{\sqrt{3}}\right)+\cos \frac{2 \pi}{a}\left(x-\frac{y}{\sqrt{3}}\right)\right. \\
& \left.+\cos \frac{2 \pi}{a}\left(\frac{2 y}{\sqrt{3}}\right)\right] .
\end{aligned}
$$

The distance $a$ between two maxima is the only geometrical parameter and it just sets the length scale of the problem, while $V_{\max }$ completely specifies the magnitude of the interactions with the surface. This potential has minima (with $V_{\min }=0$ ) connected by saddle points with an energy barrier $V_{s}=V_{\max } / 9$. In montmorillonite, $a \approx 5.2 \AA$. Furthermore, preliminary analysis of all-atom MD results suggests an approximate value of $V_{s} \simeq 12-15 \mathrm{~kJ} \mathrm{~mol}^{-1}$. A crystallographic unit cell is defined by 
(a) $\beta V_{s}=0.1$

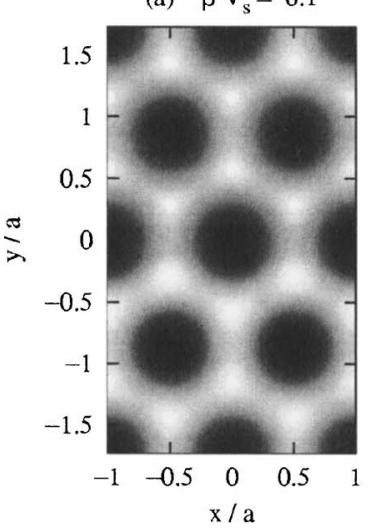

(b) $\beta V_{s}=1.0$

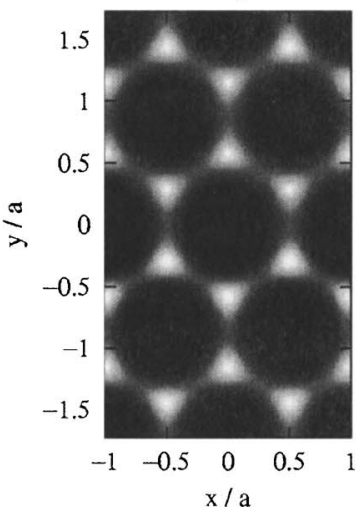

(c) $\beta \mathrm{V}_{\mathrm{s}}=2.0$

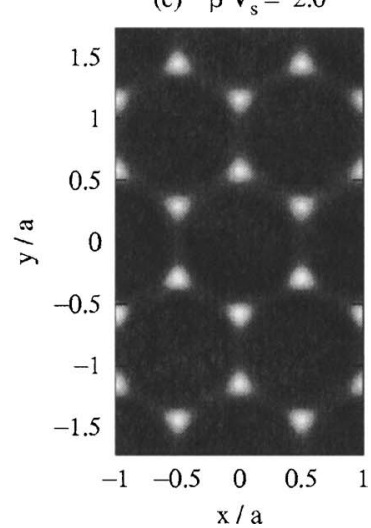

FIG. 1. Equilibrium profile in the potential (8) for $\beta V_{s}=0.1$ (a), 1.0 (b), and 2.0 (c). The gray scale code goes from black (density minima) to white (density maxima) for each figure; a given gray level does not correspond to the same density on two different figures.

$$
(x, y) \in[n a,(n+1) a] \times[m a \sqrt{3},(m+1) a \sqrt{3}],
$$

with $n$ and $m$ as integers. Each cell has an area of $a^{2} \sqrt{3}$ and contains two maxima, four minima, and six saddle points. As an example, the equilibrium density-proportional to $\exp (-\beta V(x, y))$-is shown in Fig. 1 for three values of $\beta V_{\max }=V_{\max } / k_{B} T$, corresponding to $\beta V_{s}=0.1,1.0$, and 2.0. Four cells are represented $(2 \times 2)$. For a barrier $V_{s}$ between minima larger than $k_{B} T$, the definition of distinct "sites" is quite natural, but this partition is less obvious for weaker interactions with the surface.

In principle, it would be desirable to extract the effective interaction between the solute and the surface from the potential of mean force obtained by MD simulations. However, we feel there are at least two reasons for using instead-at least as a first step - a simplified model potential. The first reason is that the time needed to obtain sufficient averages can be very long, and the resulting potential can be "noisy." The second reason is that we want to study more generally the influence of this interaction (or equivalently, the temperature), precisely by avoiding to repeat MD simulations for each temperature. Using a model potential will allow to identify trends, but of course the precise details of the interactions will be lost. Although this approach neglects the temperature dependence of the potential of mean force and of the friction, this neglect is apparently not too crude an approximation, because the model captures the basic physics of the phenomenon and will be shown to correctly reproduce the activated hopping process observed in MD simulations.

In the high friction limit $\gamma \rightarrow+\infty$, the ion velocity relaxation is fast so that the Fokker-Planck equation (4) leads via a multiple time-scale expansion to the Smoluchowski equation $^{22}$ as follows:

$$
\partial_{t} \tilde{f}(\mathbf{x}, t)=D_{0} \nabla \cdot(\nabla \tilde{f}(\mathbf{x}, t)+\beta \tilde{f}(\mathbf{x}, t) \nabla V(\mathbf{x})),
$$

where $D_{0}$ is given by (7) and $\tilde{f}(\mathbf{x}, t)$ is the position dependent part of $f(\mathbf{x}, \mathbf{v}, t)=\tilde{f}(\mathbf{x}, t) f^{M B}(\mathbf{v})$, the velocity dependent part $f^{M B}(\mathbf{v})$ being equal to a Maxwell distribution at all times. Within this regime the diffusion coefficient $D_{\text {app }}$ of the confined ions can be obtained from ${ }^{24,25}$

$$
D_{\text {app }}=D_{0}-\frac{D_{0}^{2}}{2 k_{B}^{2} T^{2}} \int_{0}^{+\infty}\langle\mathbf{F}(t) \cdot \mathbf{F}(0)\rangle d t,
$$

with $\mathbf{F}(t)=-\nabla V(\mathbf{x})=-\nabla V(x, y)$ the instantaneous force acting on the particle. Then $D_{\text {app }} / D_{0}$ does not depend on $D_{0}$ (the integral is proportional to $1 / D_{0}$, because the relaxation time is $a^{2} / D_{0}$, with $a$ the characteristic length of the external potential variations). This Smoluchowski limit is the common level of modeling of transport in bulk electrolyte solutions. ${ }^{26}$ It allows the well-known Debye-Huckel-Onsager microscopic approach to be recovered.

\section{B. From meso to two-state level}

We are now in a position to derive the two-state model described in Sec. II A from the mesoscopic description just presented. This derivation proceeds in three steps: (1) determination of the distribution constant $K_{d}$ from the apparent mobility, (2) characterization of the two states from the equilibrium distribution, and (3) determination of the exchange rates between these two states. In this section, the details of these three steps are given.

\section{Distribution constant $K_{d}$}

As explained in Sec. II A, the two-state model is defined by a chemical exchange equilibrium between mobile and adsorbed species. The distribution constant $K_{d}$ is defined by Eq. (2), and its value is determined from the measure of the apparent mobility of the ions in the external potential $V(x, y)$ (see Sec. IV A 1). The mobile fraction is

$$
\frac{\mu_{\mathrm{app}}}{\mu_{0}}=f_{m}=1-f_{a}=\frac{1}{1+K_{d}} .
$$

We thus have access, for each value of $\beta V_{\max }$, to the value of the distribution constant as follows:

$$
K_{d}=\frac{1}{f_{m}}-1=\frac{\mu_{0}}{\mu_{\text {app }}}-1 .
$$

These quantities are functions of $\beta V_{\max }$, as further elaborated in Sec. V A.

\section{Characterization of the two states}

The knowledge of the fraction of $f_{a}$ (or $K_{d}$ ) is then used to specify the nature of the two states. Although the defini- 
tion of the two states is to a certain extent arbitrary, we intuitively expect the bound ions to be located near the attractive regions on the surface, that is, on the equilibrium profile near the minima of $V(x, y)$, where the density is higher. Therefore we will define the border $\mathcal{F}$ between "adsorbed" and mobile states so that the fraction of ions with an equilibrium density larger than that on the border $\left(\rho_{\mathcal{F}}\right)$ is equal to $f_{a}$. The fraction of ions with a density lower than that on the border is automatically $f_{m}$.

$$
\begin{aligned}
& f_{a}=\frac{\int_{\rho} \mathrm{eq}_{(x, y) \geqslant \rho_{\mathcal{F}}} \rho^{\mathrm{eq}}(x, y) d x d y}{\int_{\rho}^{\mathrm{eq}(x, y) \geqslant \rho_{\mathcal{F}}} \rho^{\mathrm{eq}}(x, y) d x d y+\int_{\rho^{\mathrm{eq}}(x, y) \leqslant \rho_{\mathcal{F}}} \rho^{\mathrm{eq}}(x, y) d x d y} \\
& =\frac{N_{a}}{N_{a}+N_{m}},
\end{aligned}
$$

where $N_{a}\left(N_{m}\right)$ is the number of adsorbed (mobile) ions per unit surface. With such a definition, the border is an isodensity curve, and thus an isopotential $\left[\right.$ remember that $\rho^{\text {eq }}$ $\propto \exp (-\beta V)$, where $V$ may be a function of the density itself if self-consistent interactions are accounted for]. The border is the reunion of isopotential curves, centered on the minima of $V(x, y)$, where the sites are expected to be. There is no $a$ priori reason to exclude the possibility for these curves to be connected, but anticipating the result we can say that they are in fact disconnected, whatever the magnitude of $\beta V_{\max }$, although the precise location of the border does depend on the latter, at least in a certain range of values. This delimitation between mobile and bound ions is not without resemblance with the Fermi surface of a solid (in momentum space), because it is based on a partitioning in the energy space. In our classical case, however, the potential is defined as a function of the real-space coordinates, which allows a localization in real space.

\section{Exchange rates}

From the definition of the border, we compute the outgoing flux through the border, which is interpreted in the two-state model as the number of particles per cell switching from the bound to the mobile state per unit time as follows:

$$
j_{\text {out }}=\int_{\mathcal{F}} \int_{\mathbf{v} \cdot d \mathbf{n}>0} f(\mathbf{x}, \mathbf{v}, t) \mathbf{v} \cdot d \mathbf{n} d \mathbf{v},
$$

where $d \mathbf{n}=d l \mathbf{n}$, with $d l$ the infinitesimal length of the border and $\mathbf{n}$ is the outgoing unit normal to the border. At equilibrium, it is of course compensated by the incoming flux $j_{\text {in }}$, that is, in the two-state model by the reverse reaction.

The integral can be performed analytically. Indeed, the equilibrium probability distribution is the product of the equilibrium density by a Maxwellian distribution for the velocity as follows:

$$
f^{\mathrm{eq}}(\mathbf{x}, \mathbf{v})=\rho^{\mathrm{eq}}(\mathbf{x}) \times \frac{m}{2 \pi k_{B} T} e^{-m \mathbf{v}^{2} / 2 k_{B} T} .
$$

The normalization factor corresponds to a two-dimensional velocity space. Along the border, the density is constant, equal to $\rho_{\mathcal{F}}$, and can be factorized. Furthermore, the integral over the velocity half-plane $(\mathbf{v} \cdot d \mathbf{n}>0)$ does not depend on position and can be performed analytically, with the result

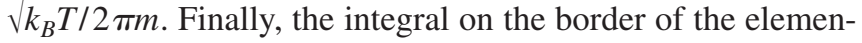
tary length is just the length of the contour $l_{\mathcal{F}}$ (again, per cell, that is, around the four minima). The final result for the flux is thus

$$
j_{\text {out }}=\rho_{\mathcal{F}} l_{\mathcal{F}} \sqrt{\frac{k_{B} T}{2 \pi m}}=\rho_{\mathcal{F}} l_{\mathcal{F}} \frac{v_{T}}{\sqrt{2 \pi}},
$$

where we have introduced the thermal velocity $v_{T}=\sqrt{k_{B} T / m}$. The flux is calculated from (17) using the values of $\rho_{\mathcal{F}}$ and $l_{\mathcal{F}}$ determined numerically via the apparent mobility route explained above.

In terms of the two-state model, the adsorption/ desorption exchange described by Eq. (1) determines the evolution of populations as follows:

$$
\partial_{t} N_{a}=-\partial_{t} N_{m}=k_{+} N_{m}-k_{-} N_{a} .
$$

The chemical relaxation time is $\tau_{\chi}=1 /\left(k_{+}+k_{-}\right)$, and of course, $K_{d}=k_{+} / k_{-}$. The link between continuous and twostate representations is provided by the identification between $j_{\text {out }}$ and $k_{-} N_{a} A_{\text {cell }}$, where $A_{\text {cell }}=a^{2} \sqrt{3}$ is the area of the unit cell. Similarly, $j_{\text {in }}=k_{+} N_{m} A_{\text {cell }}$ and at equilibrium both fluxes are equal as follows:

$$
k_{+}=K_{d} k_{-}=\frac{\rho_{\mathcal{F}} l_{\mathcal{F}} v_{T}}{\sqrt{2 \pi} A_{\text {cell }} N_{m}} .
$$

\section{LATTICE FOKKER-PLANCK RESULTS}

\section{A. Method}

\section{Apparent mobility determination}

The apparent mobility is measured as follows. For each value of the potential maximum $\beta V_{\max }$, the equilibrium solution of (4) is first determined numerically, and the resulting moments $\rho$ and $\mathbf{j}$ [Eqs. (5) and (6)] are compared with the analytical results [moments of Eq. (16)]. Then, starting from this equilibrium situation, a force $m a_{x}$ is applied along the $x$ direction and the average steady state flux $j_{x, \text { st }}$ is sampled. For low values of $a_{x}$, one observes a linear relationship between $j_{x, \text { st }}$ and $\rho a_{x}$. The proportionality constant defines the apparent mobility $\mu_{x, \text { app }}$. The same procedure with an applied

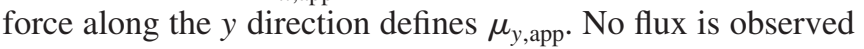
(see below for details) in the direction normal to the applied field (the medium is overall isotropic). Note that in the absence of interactions with the surface $\left(\beta V_{\max }=0\right)$, the mobility is isotropic and its value is simply $\mu_{0}=1 / \gamma$. In fact, in a potential with hexagonal symmetry, the mobility tensor is still isotropic (see below), and the value of $\mu_{\mathrm{app}}=\mu_{x \text {,app }}$ $=\mu_{y, \text { app }}$, lower than $\mu_{0}$, is interpreted as explained in Sec. I as the result of adsorption of a fraction $f_{a}$ of the ions [Eq. (12)].

\section{Computational details}

The Fokker-Planck equation is solved with the D2Q9-

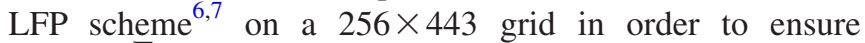
$L_{y} / L_{x}=\sqrt{3}$. On this grid, four unit cells $(2 \times 2)$ are simulated (see Fig. 1). Therefore the lattice unit length is $\Delta x=a / 128$. The lattice unit time is then fixed by the relation $v_{T}^{2}=(1 / 3)$ $\times(\Delta x / \Delta t)^{2}=k_{B} T / m$, where the factor $1 / 3$ is a characteristic feature of the D2Q9 lattice. For $\mathrm{Cs}^{+}\left(m=0.133 \mathrm{~kg} \mathrm{~mol}^{-1}\right)$ at 
room temperature, $v_{T} \sim 136 \mathrm{~m} \mathrm{~s}^{-1}$. The external potential is introduced directly as the acceleration $\mathbf{F} / m=-\nabla V / m$. Periodic boundary conditions were used in both directions. Finally, the simulation is performed for a value of the friction corresponding to the diffusion coefficient of mobile ions such that $D_{0}=v_{T}^{2} / \gamma$; that is, $\gamma \Delta t=D_{\text {latt }} / D_{0}$, with $D_{\text {latt }}=(1 / 3)$ $\times \Delta x^{2} / \Delta t$ the lattice unit diffusion coefficient. Unless otherwise stated, the simulations where done for $\gamma \Delta t=0.1$; that is, a mobility $\mu_{0}=10$ (in reduced units) in the absence of interactions with the surface. This choice corresponds for $\mathrm{Cs}^{+}$in montmorillonite $(a \approx 5.2 \AA)$ to a diffusion coefficient at room temperature of $D_{0} \sim 3.10^{-9} \mathrm{~m}^{2} \mathrm{~s}^{-1}$.

A second-order time integration scheme was used, and we assumed that the validity of the scheme, demonstrated for a homogeneous applied force, ${ }^{7}$ could be extended to the case of a slowly varying inhomogeneous force field. The use of a large number of grid points for each cell was chosen for that purpose. Equilibration runs started from a homogeneous distribution of the ions with density $\rho_{0} \times \Delta x^{2}=1.0$. The numerical value of the density is not important since the ions are treated as independent (ideal gas in an effective potential). Note that this particular value is in fact much higher than the experimental one (approximately 0.75 ion per unit cell, that is, $\left.\rho_{0} \times \Delta x^{2} \approx 2.6 \times 10^{-5}\right)$. The duration of the run was $3 \times 10^{3}$ time steps. The resulting density coincides with the Boltzmann law, but the velocity field is nonzero: even for weak external fields $\left(\beta V_{\max } \ll 1\right)$, a residual velocity is observed whose magnitude increases with increasing external field. In reduced units, the average residual velocity is of order $10^{-9}$ for $\beta V_{\max } \sim 0.1$, and of order $10^{-4}$ for $\beta V_{\max }$ $\sim 20$, although the stationary state is already reached. Since these values are small, much smaller at least than the final velocity in the presence of an applied field (see below and Fig. 2), we can consider that the equilibrium solution (16) of the Fokker-Planck (FP) equation is reached. This residual value could arise from the discretization of the potential on a lattice or to a lack of accuracy of the scheme for an inhomogeneous force field.

For each $\beta V_{\max }$ value, the border described in Sec. III B 2 was determined from the numerical value of $f_{m}$ by integrating "à la Lebesgue," that is, by density "slices," starting from the lowest density until the integral is equal to $f_{m}$ $\times \rho_{0}$. For the contour to be well defined, one needs to have enough grid points in each density slice, and the number of slices $N_{s}$ should be chosen accordingly. A compromise between the accuracy of the integral and that of the definition of the border must be found. The latter has a strong influence on the calculated exchange rate (see Sec. III B 3). $N_{s}=50$ was found to be a good compromise, except for the lowest values of $\beta V_{\max }$. In order to increase the resolution of the border, the numerically determined curve was fitted to an isopotential curve using the GNUPLOT software. ${ }^{27}$ The density on the border and its length were then numerically determined to calculate the flux and exchange rates.

\section{Results}

For the mobility measurements, applied accelerations ranging from $10^{-4}$ to $10^{-2}$ (in lattice units) were used. The

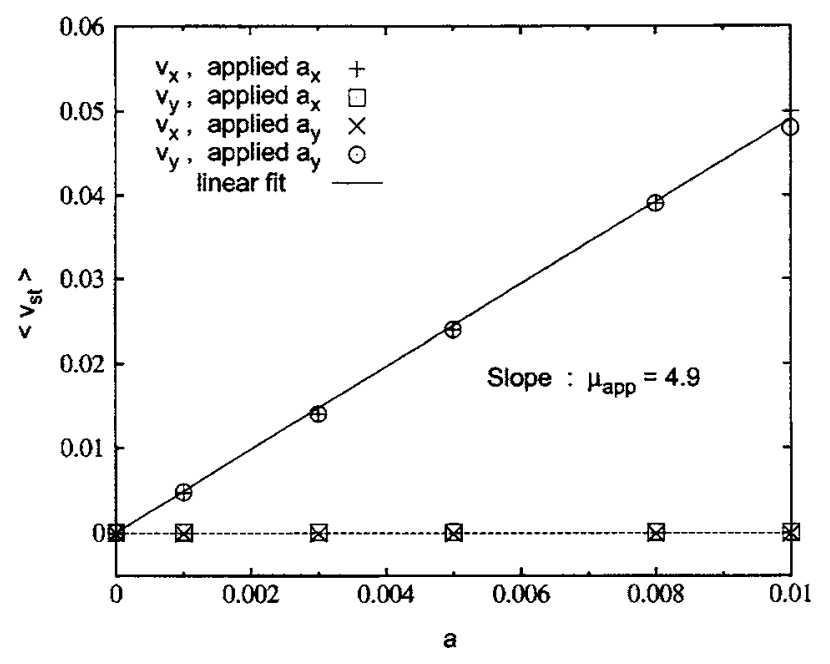

FIG. 2. Steady-state velocity as a function of the applied field for an interaction with the surface characterized by $\beta V_{\max }=12\left(\beta V_{s}=4 / 3\right)$. The slope of the linear fit is the apparent mobility $\mu_{\text {app}}$, which is independent of the considered direction. The residual velocity in the direction normal to the applied field is negligible. The units are $\Delta x / \Delta t^{2}$ for $a, \Delta x / \Delta t$ for $v_{\text {st }}$, and $\Delta t$ for $\mu_{\mathrm{app}}$.

average velocity ( $x$ and $y$ components) is computed after $3 \times 10^{3}$ time steps, which is enough to ensure that the steady state is reached,

$$
\left\langle u_{x, \mathrm{st}}\right\rangle=\frac{\int_{\text {cell }} \rho(x, y) u_{x}(x, y) d x d y}{\int_{\text {cell }} \rho(x, y) d x d y}
$$

and a similar expression for $\left\langle u_{y \text {,st }}\right\rangle$. The linear dependence of $\left\langle u_{x, \mathrm{st}}\right\rangle$ on $a_{x}$ is illustrated in Fig. 2 for an interaction with the surface characterized by $\beta V_{\max }=12$. The slope of the fit curve defines the apparent mobility. It is also obvious from this figure that the average velocity normal to the applied field is negligible, that is, of the order of the residual velocity in the absence of any applied field. This justifies the above statement of an isotropic mobility tensor and the definition of a single apparent mobility $\mu_{\text {app }}$. The comparison of the values of $\mu_{x, \text { app }}$ and $\mu_{y, \text { app }}$ gives an estimate of the error made in the determination of $\mu_{\text {app }}$.

The mobility was determined for values of $\beta V_{\max }$ ranging from 0 to $27\left(\beta V_{s}\right.$ between 0 and 3$)$. The results are represented in Fig. 3. As expected, the mobility decreases for increasing affinity for the surface (increasing $\beta V_{\max }$ ). This is consistent with the two-state picture, in which $\mu_{\text {app }} / \mu_{0}$ is simply the fraction of mobile ions $f_{m}$. Two regimes are observed that correspond roughly to $\beta V_{s}<1$ and $\beta V_{s}>1$. Their significance will become more obvious when interpreted in terms of $K_{d}$. Let us first mention that the fraction of mobile ions does not depend on the solvent friction (that is, on their "free" mobility $\mu_{0}$ ), at least in this (high) friction range. This result is illustrated on the inset of Fig. 3, where the data are shown for $\gamma=0.1$ and 0.2 (in lattice units). This justifies $a$ posteriori that the exact value of the friction was not necessary to define the two states, because only the reduced mobility is involved. More insight in the meaning of such a diffusion curve can be obtained by comparing these results to that of simpler existing approximations. 


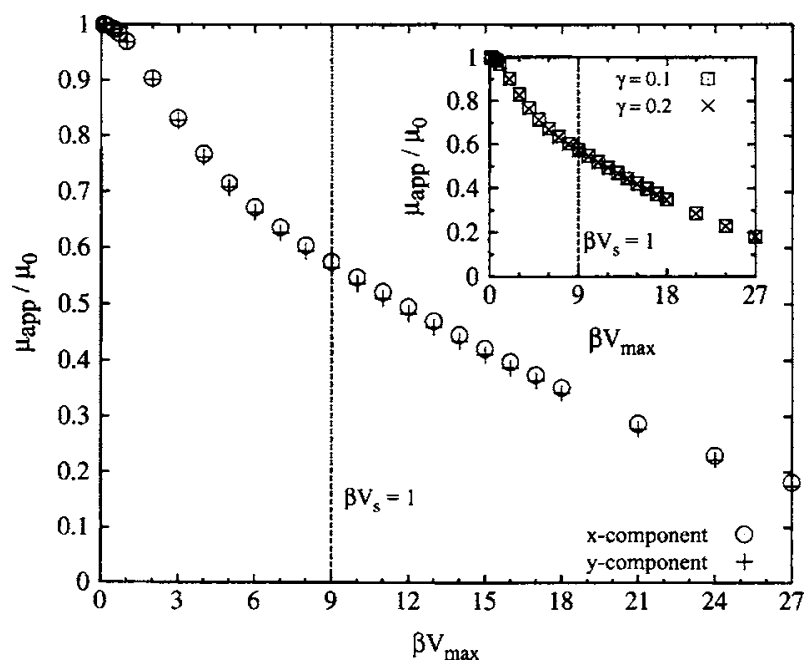

FIG. 3. Rescaled apparent mobility $\mu_{\text {app }} / \mu_{0}$ as a function of $\beta V_{\max }$. The vertical line indicates where the thermal energy is equal to the potential at the saddle point (barrier separating two minima). The values obtained for the $x$ and $y$ components are shown for a friction $\gamma \Delta t=0.1$. The mobility is seen to be isotropic in the honeycomb lattice. The inset shows the values of the $x$ mobility for two frictions, $\gamma \Delta t=0.1$ and 0.2 ; in this high friction range, $\mu_{\text {app }} / \mu_{0}$ is friction independent (Smoluchowski regime). As expected, the mobility decreases for increasing affinity for the surface. This is consistent with the two-state picture, in which $\mu_{\text {app }} / \mu_{0}$ is simply the fraction of mobile ions.

\section{B. Comparison with various approximations}

We now compare the apparent mobility determined by direct measurement via the lattice Fokker-Planck scheme with three other methods, namely, (1) a low-potential approximation first demonstrated by Bagchi, ${ }^{28}$ (2) the "quasitwo-dimensional approximation," 20 and (3) the "diffusion path approximation." 20 All three approximations are applicable in the high-friction range, which is the relevant one for ionic transport in clays. Although they are of simpler use than the LFP method, they all give inappropriate results in at least one temperature range (generally at low temperature). This justifies the use of the LFP method, which may be considered to be exact for the implicit solvent model of independent ions.

\section{Perturbative treatment (low-potential approximation)}

In the liquid crystal context, a perturbative treatment of the diffusion of a particle in an external potential was introduced, in the high-friction (Smoluchowski) regime. ${ }^{28}$ This calculation is based on the following formal exact expression of the (apparent, i.e., potential dependent) diffusion coefficient:

$$
D_{P}=\frac{1}{2} \operatorname{Tr}\left[\frac{1}{2} \nabla_{\mathbf{k}} \nabla_{\mathbf{k}} \lambda(\mathbf{k})\right]_{\mathbf{k}=0},
$$

where in the $\mathbf{k} \rightarrow 0$ limit,

$$
\lambda(\mathbf{k})=D_{0} k^{2}-i D_{0} \mathbf{k} \cdot \frac{\int d \mathbf{x} \exp (-\beta V) \nabla \xi_{\mathbf{k}}(\mathbf{x})}{\int d \mathbf{x} \exp (-\beta V)},
$$

where $i^{2}=-1$, and $\xi_{\mathbf{k}}$ is a solution of the partial differential equation as follows:

$$
-\nabla \cdot\left(\exp (-\beta V) \nabla \xi_{\mathbf{k}}\right)=i \mathbf{k} \cdot \nabla \exp (-\beta V) .
$$

Due to the periodicity of the system, it is convenient to work in reciprocal space. The Fourier components are then defined from

$$
\begin{aligned}
& E(\mathbf{q})=\frac{1}{\Delta} \int d \mathbf{x} \exp (-\beta V) e^{-i \mathbf{q} \cdot \mathbf{x}} \\
& F(\mathbf{q})=\frac{1}{\Delta} \int d \mathbf{x} \xi_{\mathbf{k}} e^{-i \mathbf{q} \cdot \mathbf{x}},
\end{aligned}
$$

where $\mathbf{q}$ is a reciprocal lattice vector and $\Delta$ is the volume of the crystallographic cell. A perturbative solution scheme for $F(\mathbf{q})$ can be derived. ${ }^{28}$ At high temperature, the first-order term reads

$$
F(\mathbf{q})=\frac{\mathbf{k} \cdot \mathbf{q} \beta V(\mathbf{q})}{q^{2}},
$$

where $V(\mathbf{q})$ are the Fourier components of $V(\mathbf{x})$ in the reciprocal space defined similarly to (24) and (25). Substitution of (26) in Eqs. (21) and (22) leads to

$$
\frac{D_{P 1}}{D_{0}}=1+\sum_{\mathbf{q}} \frac{\beta V(\mathbf{q}) E(-\mathbf{q})}{2 E(\mathbf{0})} .
$$

An alternative expression for the apparent diffusion coefficient can be derived more directly by writing

$$
F(\mathbf{q})=-\frac{\mathbf{k} \cdot \mathbf{q} E(\mathbf{q})}{q^{2} E(\mathbf{0})}
$$

so that

$$
\frac{D_{P 2}}{D_{0}}=1-\sum_{\mathbf{q}} \frac{E(\mathbf{q}) E(-\mathbf{q})}{2 E^{2}(\mathbf{0})} .
$$

Equations (27) and (29) are equivalent to the solutions proposed in Ref. 28 in the context of the liquid-crystal interface. The variations of $D_{P 1}$ and $D_{P 2}$ with $\beta V_{\max }$ are reported in Fig. 4 (long and short dashed lines, respectively). At high temperature $\left(\beta V_{\max } \ll 1\right)$, the perturbative results compare favorably with the LFP results. At low temperature, however, the diffusion coefficients predicted by Eqs. (27) and (29) are too low and even become negative. The domain of validity of approximation (29) is larger than that of (27). The breakdown of the perturbative treatment in this regime is not unexpected.

\section{Quasi-2D approximation}

A second type of approximation was introduced by AlaNissila and Ying ${ }^{17,18}$ for the study of surface diffusion. Later named quasi-2D approximation by Caratti et al., ${ }^{20}$ it is equivalent to an average of the $x$ mobility $(y)$ over the $y$ direction $(x)$, that is,

$$
\mu_{Q 2, x}=\left\langle\mu_{x}\right\rangle_{y} .
$$

The $x$ mobility corresponds in that case to the mobility of particles evolving along one-dimensional trajectories (in the $x$ direction), and the average is performed over parallel trajectories, at variance with the LFP approach where the aver- 


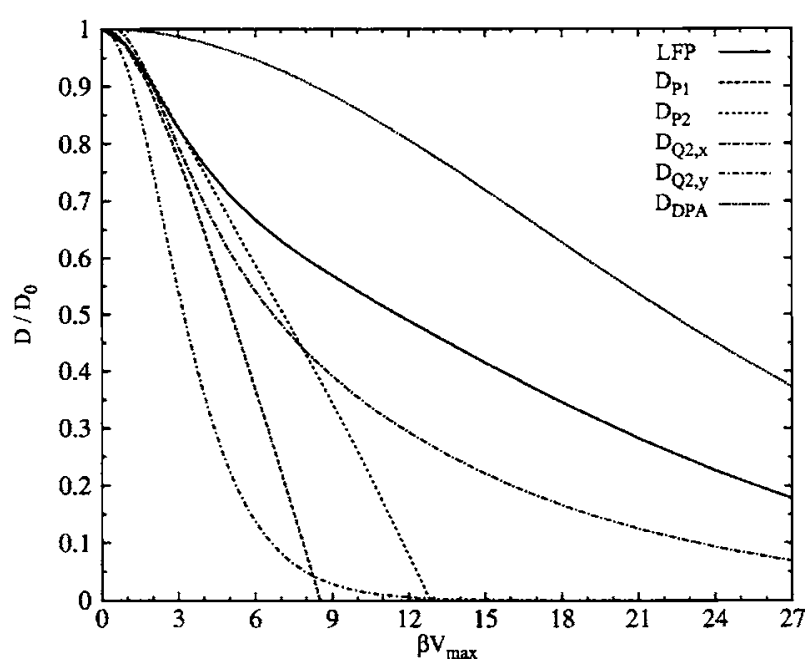

FIG. 4. Rescaled apparent mobility $\mu_{\mathrm{app}} / \mu_{0}$, as a function of $\beta V_{\max }$, obtained with the perturbative treatments of Eqs. (27) and (29) (dashed line), the quasi-2D approximation (dotted-dashed line, $x$ and $y$ components) and the diffusion path approximation (dotted line). The LFP result (solid line) is also shown (same as Fig. 3).

age is done over a unit cell without any assumption on the trajectory followed by the particles. In the case of an asymmetric unit cell $\left(L_{x} \times L_{y}\right)$, the result is ${ }^{19,29}$

$$
\frac{D_{Q 2, x}}{D_{0}}=L_{x}^{2} \times \frac{\int_{0}^{L_{y}} d y\left[\int_{0}^{L_{x}} d x \exp [\beta V(x, y)]\right]^{-1}}{\int_{0}^{L_{y}} d y \int_{0}^{L_{x}} d x \exp [-\beta V(x, y)]}
$$

and a similar result for the $y$ mobility.

The results are reported in Fig. 4. The first observation is that the quasi-two-dimensional approximation predicts a strong anisotropy $\left(D_{Q 2, y} \ll D_{Q 2, x}\right)$, in contradiction with both the MD results for ionic diffusion in clays and the general behavior on a honeycomb lattice. ${ }^{23}$ The failure in this particular case was already expected in Ref. 20, where it was recognized that " the quasi-2D approximation is expected to give good results when the minima and saddle points lie on straight lines (...); in a honeycomb lattice, this is not the case and one could expect a worse agreement between the quasi-2D approximation and the exact data." Indeed, the trajectories followed by the particles (roughly connecting the minima via the saddle points) are broken lines in that case. The second feature of the quasi-2D approximation is that it underestimates the diffusion coefficient (the LFP result being regarded as exact). This follows from the approximation that the trajectories followed by the ions are straight lines that will necessarily cross regions of high potential (this lowers the mobility). These regions are in fact never explored by the particles. An Arrhenius behavior $D \propto \exp \left(-\beta E_{a}\right)$ is observed at low temperature, but with an activation energy $E_{a}$ that is direction dependent and larger than the difference $V_{s}$ between the minima and the saddle points (result not shown). These are direct consequences of the above-mentioned limitations of the quasi-2D approximation for the honeycomb lattice.

\section{Diffusion path approximation}

The last approximation to be tested is the diffusion path approximation (DPA). In this approximation, the 2D problem is reduced to a one-dimensional (1D) situation by assuming that the trajectories followed by the particles in the $2 \mathrm{D}$ potential are restricted to the minimum-energy paths (MEPs). This approximation is expected to be reasonable only at low temperature, where the trajectories followed in the real situation are indeed close to the MEP. In the case of a periodic potential where these are straight lines, the $1 \mathrm{D}$ potential is also periodic, and the classic result ${ }^{19,20}$

$$
\frac{D_{\mathrm{DPA}}}{D_{0}}=\frac{L^{2}}{\int_{0}^{L} d s \exp [\beta \mathcal{V}(s)] \int_{0}^{L} d s \exp [-\beta \mathcal{V}(s)]}
$$

applies, where the distance between two minima was denoted by $L$ and the potential $\mathcal{V}(s)$ is the restriction of $V(x, y)$ to the (one-dimensional) minimum-energy path. In our case, the MEPs are not straight lines. However, since the derivation of (32) is based on the mean jump time between two minima, ${ }^{30}$ the same result is recovered for the reduced diffusion coefficient $D_{\mathrm{DPA}} / D_{0}$ in this case too, without any geometric correction factor (that relates the jump time to the diffusion coefficient).

The results are represented in Fig. 4. The DPA overestimates the diffusion coefficient over the whole temperature range. This can be understood as follows. In the case of true two-dimensional diffusion, the particles explore the whole surface with high probability, resulting in a less efficient diffusion than in the situation underlying the DPA. Indeed, in the latter case the particles are restricted to one-dimensional paths (which are relevant for high-potential barriers) on the $2 \mathrm{D}$ surface. It is a well-known fact that reducing the dimensionality increases the efficiency of the diffusion process, and examples, particularly in biological systems, are numerous. ${ }^{31}$ Let us, for example, mention the mobilization of reactants at the (2D) surfaces of [three-dimensional (3D)] cells, and the DNA binding molecules that can find genes on a DNA strand very efficiently by first binding to the DNA, then sampling it via a $1 \mathrm{D}$ motion (instead of sampling a 3D space). Although these systems are infinitely more complicated than the one studied here, they illustrate the concept of diffusion efficiency enhancement by reduction of the dimensionality. A good feature of the DPA is that it predicts at low temperature $\left(\beta V_{\max } \gg 1\right)$ an Arrhenius behavior with an activation energy of $E_{a}=V_{s}$ as expected (result not shown). The prefactor is, however, overestimated, leading to too large a diffusion coefficient.

These three simple approximations lead to inaccurate results and are unable to reproduce correctly the apparent diffusion coefficient in a hexagonal (honeycomb) lattice over the whole temperature range studied. This justifies the use of a more elaborate method, namely, lattice Fokker-Planck, to determine the apparent mobility. From this determination, we are now able to construct the two-state model as described in Sec. III.

\section{EXPLICIT COARSE-GRAINING: CONSTRUCTION OF THE TWO-STATE MODEL}

\section{A. Distribution constant $\boldsymbol{K}_{\boldsymbol{d}}$}

We first translate the apparent mobility determined in Sec. IV A in terms of the distribution constant $K_{d}$, calculated 


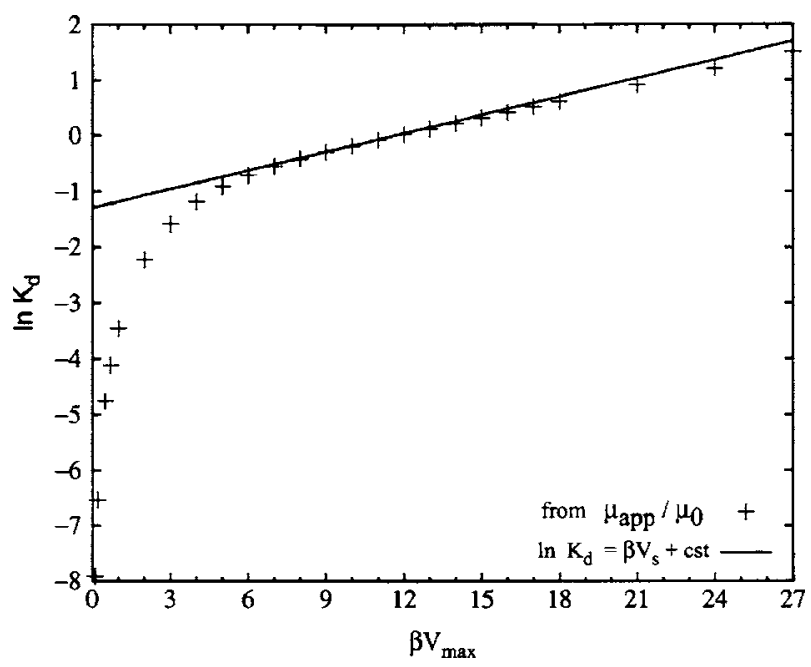

FIG. 5. Distribution constant $K_{d}$, as a function of $\beta V_{\max }$, on a logarithmic scale. At high interaction potentials (low temperature), $\ln K_{d}$ is a linear function of $\beta V_{\max }$

from (13). The results are represented in Fig. 5 on a logarithmic scale. The first observation is that the adsorption equilibrium is, as expected, displaced towards the adsorption reaction upon increasing the affinity for the surface, as reflected by the increase in $K_{d}$ with $\beta V_{\max }$. Furthermore, one distinguishes two regimes: at high $\beta V_{\max }, \ln K_{d}$ is a linear function of $\beta V_{\max }$, while it is not the case for weak interaction with the surface. More precisely, the slope of the linear part corresponds to $\ln K_{d}=\beta V_{s}+\mathrm{B}$, with $V_{s}=V_{\max } / 9$ the energy difference between the minima and the saddle points, and $B \sim-1.35$. The crossover between the two regimes corresponds to the shift from a picture of Brownian, liquidlike diffusion at high temperature $\left(k_{B} T \gg V_{s}\right)$ to an activated hopping process at low temperature $\left(k_{B} T \ll V_{s}\right)$, consistent with the appearance of well-localized sites (see Fig. 1). The activation energy of the hopping process is simply $V_{s}$. Interestingly, all approximations presented in Sec. IV B predict a crossover between Brownian diffusion and activated hopping, but with a wrong activation energy (except for the DPA, which by construction gives a correct $E_{a}$ and only overestimates the prefactor).

The significance of $V_{s}$ as an energy gained by the ion by interacting with the surface is quite natural, although the apparent mobility route would rather suggest an interpretation in terms of activation energy. The meaning of the shift $B$ is less obvious. A possible interpretation would be the following. The adsorption reaction also involves the trapping site and is described as the chemical equilibrium,

$$
\text { mobile }+ \text { site } \leftrightarrows \text { adsorbed }
$$

The thermodynamic equilibrium constant of this reaction is

$$
K_{\text {trapping }}=\frac{a_{\text {ads }}}{a_{\text {mobile }} a_{\text {site }}}=\frac{[\text { ads }]}{[\text { mobile }][\text { site }]},
$$

with $a_{i}$ the activity of species $i,[i]$ its surface concentration, and where ideality has been assumed. The last equation can be rewritten as

$$
K_{\text {trapping }}=\frac{K_{d}}{[\text { site }]}
$$

or in terms of the free energy of trapping as follows:

$$
-\beta \Delta F_{\text {trapping }}=\ln K_{\text {trapping }}=\ln K_{d}-\ln [\text { site }] .
$$

By identification, this suggests the following:

$$
\begin{aligned}
& \beta \Delta F_{\text {trapping }}=-\beta V_{s}, \\
& B=\ln [\text { site }] .
\end{aligned}
$$

The site surface concentration would then determine the value of $B$. This value is consistent with the fraction of the surface corresponding to a potential energy lower than $V_{s}$. Indeed, it is easily shown to be

$$
\alpha=\frac{\int_{V(x, y) \leqslant V_{s}} d x d y}{\int_{\text {cell }} d x d y}=\frac{1}{4}
$$

and $\ln \alpha \sim-1.39$. Therefore it is tempting to interpret the site concentration as $\alpha$. This thermodynamic/geometric route can be further explored. In particular, the last result can be recovered if the system is modeled by a simple two-region potential, separated by an energy difference $V_{s}$. In that case, the calculation of the partition function shows that the mass action law reads

$$
\ln K_{d}=\ln \frac{S_{\text {adsorbed }}}{S_{\text {mobile }}}+\beta V_{s},
$$

with $S_{i}$ the surface corresponding to state $i$. Hence $\alpha$ $=\ln [(1 / 4) /(3 / 4)] \sim-1.1$.

\section{B. Characterization of the two states}

It seems natural to define the bounding sites as just explained, because no reference is made to the ions that evolve in this potential surface. However, this definition is somewhat arbitrary. Furthermore, the fraction of ions that is located at equilibrium on these geometrical sites does not in general correspond to the fraction of adsorbed ions defined in the first place by Eq. (14). Since the latter is the one that corresponds to the mobility approach, it is the relevant one for our initial purpose, that is precisely to define a two-state model consistent with the apparent mobility. We now present the results obtained as described in Sec. III B 2. The border defined by Eq. (14) is shown as a function of $\beta V_{\max }$ in Fig. $6(\mathrm{a})$. Only the border around a single minimum of $V(x, y)$ is represented. The curves shown are fits to isopotential curves of the borders determined numerically on the lattice grid (see Sec. IV A 2). The values of the corresponding potentials $V_{\mathcal{F}}$ are reported in Fig. 6(b).

Firstly, let us note that the adsorbed region is always smaller than the one defined by $V=V_{s}$, and it depends on $\beta V_{\max }$, that is, on the maximum potential (or on temperature). Thus the "natural" definition of a site might not be the most appropriate to describe the change in mobility due to the interactions with the surface. Secondly, it becomes wider with increasing potential or decreasing temperature, until it reaches a limit shape, characterized by $V_{\mathcal{F}}=\lambda V_{s}$, with $\lambda$ $\sim 1 / \sqrt{3}$. As seen in Fig. 6(b), the corresponding $V_{\mathcal{F}}$ increases 

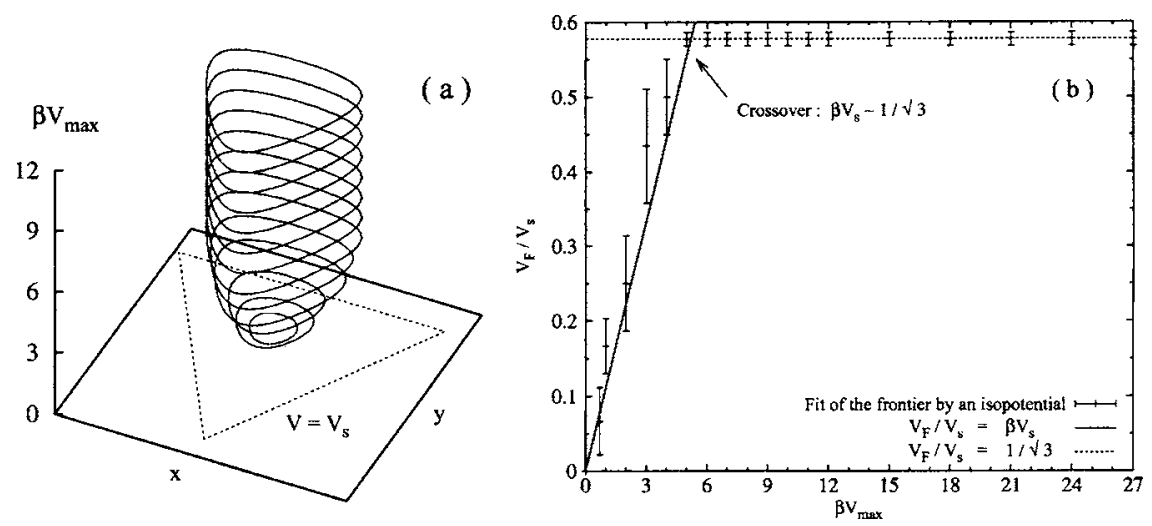

FIG. 6. (a) Frontier between the two states as a function of $\beta V_{\max }$. Only a part of the border, corresponding to one minimum of $V(x, y)$, is represented. The curve shown is a fit of the border determined numerically by an isopotential curve. The isopotential $V=V_{s}$ is also indicated (dotted line). For strong potentials, the location of the border does not depend on $\beta V_{\max }$ although $K_{d}$ and the density on the border $\rho_{\mathcal{F}}$ do. (b) Value of the potential $V_{\mathcal{F}}$ on the border as a function of $\beta V_{\max }$. At high values of the potential (or low temperature), $V_{\mathcal{F}} \sim \lambda V_{s}$ with $\lambda \sim 1 / \sqrt{3}$, while at low potential (high temperature), $\beta V_{\mathcal{F}} \sim\left(\beta V_{s}\right)^{2}$.

approximately as $\beta V_{\mathcal{F}} \sim\left(\beta V_{s}\right)^{2}$, until a crossover value of $\beta V_{s} \sim \lambda$. It is not surprising to find a crossover that involves the ratio between $V_{s}$ and the thermal energy $k_{B} T$, however, the critical value probably arises from the particular shape of the potential (its analytical form). A value lower than 1 indicates an overestimation of $K_{d}$ by defining the sites by $V$ $\leqslant V_{s}$. Finally, for high potentials (low temperatures), the definition of the border is independent of $\beta V_{\max }$, although $K_{d}, V_{\mathcal{F}}$, and $\rho_{\mathcal{F}}$ do depend on it. This justifies the notion of site for strong interactions with the surface (low temperature). A consequence for the calculation of the exchange rate is that the length of the border $l_{\mathcal{F}}$ is independent of $\beta V_{\max }$ in this range, so that the variations of $j_{\text {out }}$ are governed by that of $\rho_{\mathcal{F}}$ [see Eq. (17)].

\section{Exchange rates}

The flux through the border is now calculated from (17) with the numerical values of the density $\rho_{\mathcal{F}}$ and the contour length $l_{\mathcal{F} .} \rho_{\mathcal{F}}$ is determined by averaging the density along the border, and an estimate of the error is provided by the difference between the maximum and minimum values in the slice determining the border (see Sec. IV A 2). The results are reported in the inset of Fig. 7. The evolution of $\rho_{\mathcal{F}}$ with $\beta V_{\max }$ displays a maximum. For weak interactions, the density increases because the border is very close to the potential minimum; for very high potentials, the density is so sharply peaked that the limit border is already on the tail of the density profile.

Since the flux is proportional to the thermal velocity, the results as a function of $\beta V_{\max }$ are to be understood at fixed temperature and variable $V_{\max }$. The variations of the flux (Fig. 7) are governed by that of the density on the contour $\rho_{\mathcal{F}}$. The evolution of the adsorption/desorption rate constants with $\beta V_{\max }$ are given in Fig. 8(a) together with that of the chemical relaxation time $\tau_{\chi}=1 /\left(k_{+}+k_{-}\right)$[Fig. 8(a)]. The adsorption rate increases with increasing interaction with the surfaces, whereas the desorption rate decreases.

$\tau_{\chi}$ is always (a few times) longer than the inverse friction and displays a maximum for an intermediate value of $\beta V_{\max }$. We can compare $\tau_{\chi}$ to the characteristic diffusion time $\tau_{\text {diff }}$ defined as the time it takes an ion to diffuse over the mean interionic distance $L$ :

$$
\tau_{\text {diff }}=\frac{L^{2}}{D}=\frac{L^{2}}{v_{T}^{2}} \times \gamma
$$

In montmorillonite $L \approx 8 \AA$, leading to a ratio $\tau_{\chi} / \tau_{\text {diff }}$ $\sim 10^{-4}$. In other words, we are in a "fast exchange" regime characterized by a large number of adsorption/desorption processes during the diffusion time $\tau_{\text {diff. }}$. This is because the exchange reaction corresponds here to oscillations around the potential minima (more precisely, to the crossing of the limiting contour $V=V_{\mathcal{F}}$ ), while the diffusion requires the crossing of the saddle points, which happens much less frequently (at least for low temperature). Since these oscillations are driven by the solvent friction, it is not surprising to find $\gamma \tau_{\chi} \sim 1$. Recent studies investigated the influence of the ratio $\tau_{\chi} / \tau_{\text {diff }}$ on the frequency-dependent permittivity of

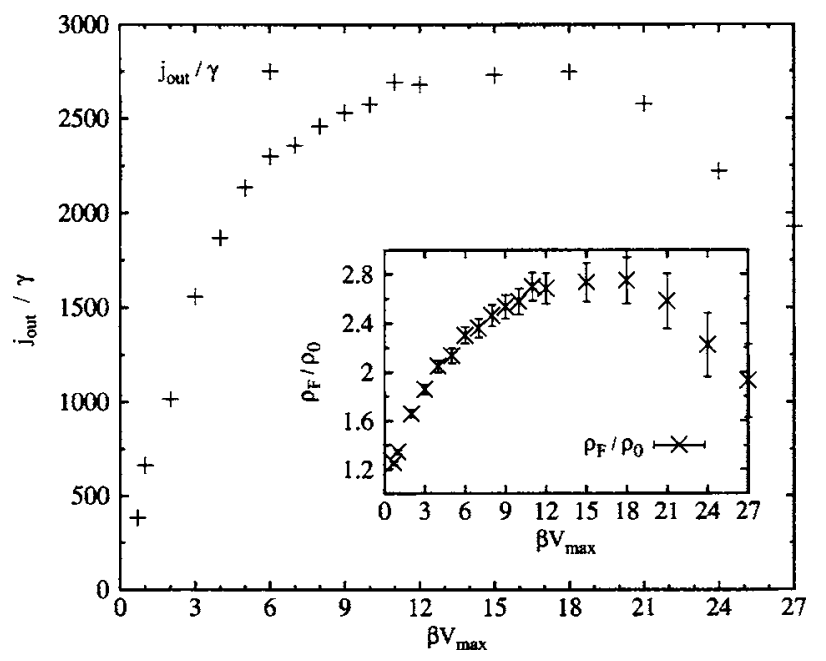

FIG. 7. Flux through the border as a function of $\beta V_{\max }$. The result is given per unit cell, scaled by the value of the friction $\gamma=0.1 \Delta t^{-1}$. The variations of $j_{\text {out }}$ are governed by that of $\rho_{\mathcal{F}}$, reported in the inset. The density is scaled by the average density $\rho_{0}$. The density shows a maximum as a function of $\beta V_{\max }$. For strong interactions with the surface, $\rho_{\mathcal{F}}$ decreases because the profile is sharply peaked around the minimum of $V$, and the border is far enough from this minimum to be on the "tail" of the peak. 

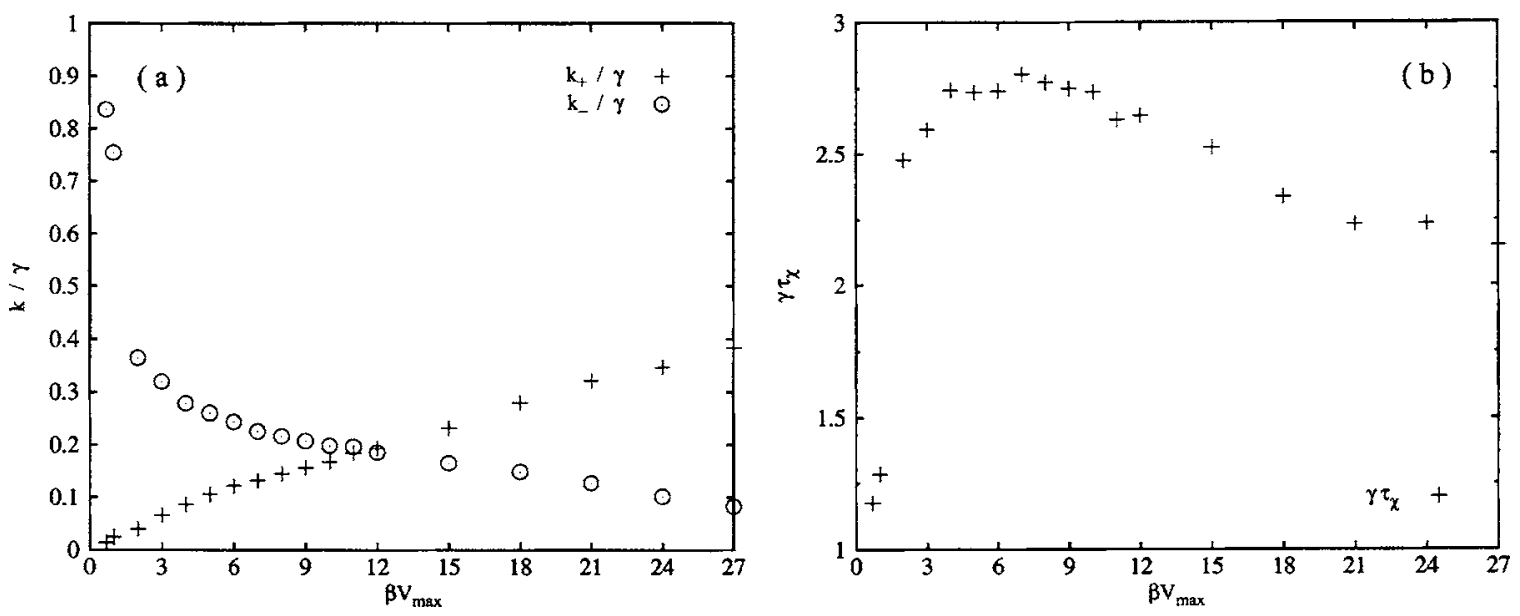

FIG. 8. (a) Adsorption (+) and desorption $(\bigcirc)$ rates as a function of $\beta V_{\max }$. The results are scaled by the value of the friction $\gamma=0.1 \Delta t^{-1}$. The ratio between both rates is simply $K_{d}$. (b) Chemical relaxation time as a function of $\beta V_{\max }$. The results are scaled by the inverse friction $\gamma^{-1}=10 \Delta t$.

clays. $^{32,33}$ The authors showed that in the fast exchange limit, only one dielectric relaxation could be observed, whereas two could be distinguished for slow exchange. However, a direct comparison is difficult, because the chemical relaxation rate was defined in a slightly different way. The influence of the bound/free equilibrium on the dielectric properties of water at the surface of biological molecules had also been established. ${ }^{34}$ In this case, the dielectric consequences of the exchange arise from the modification of the rotational properties of water (which is polar but neutral), whereas in the case of (charged) ions the dielectric behavior is affected by the change in mobility.

\section{CONCLUSION}

We have demonstrated how to completely define a twostate model from the microscopic dynamics of ions in clays. All the equilibrium $\left(K_{d}\right)$ and dynamic (adsorption/desorption rates) properties of this two-state model were studied as a function of temperature. This approach gives a microscopic basis to the definition of bound and mobile states that is consistent with the apparent mobility concept. In particular, the notion of trapping site was shown to be relevant in this context too, for sufficiently strong interactions with the surfaces (low $T$ ). Furthermore, in this regime, the diffusion is activated, in agreement with the common picture of jump diffusion.

The comparison of the lattice Fokker-Planck results for the apparent mobility with simple approximations (low potential, quasi-2D, and diffusion path approximations) illustrated the need for the use of this more elaborate method. Although the present study was done for a model external potential, which captures all the physics revealed by molecular dynamics simulations (diffusion mechanism, influence of temperature), it would be possible to adapt the same approach to the effective potential obtained from MD simulations. Furthermore, the particles were treated as independent, while self-consistent interactions between the ions could be included at this level of description within the framework of the Poisson-Boltzmann theory. Finally, only the steady-state velocity was used to determine the apparent mobility, but further information can be extracted from the transient. The latter is influenced by the friction of the solvent, and we are planning to calibrate the precise value of the friction in order to reproduce the velocity autocorrelation function of the ions from MD simulations. This will complete the coarse-graining procedure from the atomic level to the macroscopic two-state picture.

\section{ACKNOWLEDGMENTS}

One of the authors (B.R.) acknowledges the financial support of the Agence Nationale pour la Gestion des Déchets Radioactifs (ANDRA, France). Another author (B.B.) thanks DST and CSIR (India) for partial support of this work. Fruitful discussions with O. Benichou are gratefully acknowledged.

${ }^{1}$ H. Kato, M. Muroi, N. Yamada, H. Ishida, and H. Sato, Scientific Basis for Nuclear Waste Management (Materials Research Society, Pennsylvania, 2005), Vol. 18.

${ }^{2}$ ANDRA, Matériaux à Base d'Argiles Gonflantes, Référentiel Matériaux, Vol. 1 (ANDRA, Châtenay Malabry, France, 2005).

${ }^{3}$ C. Daqing and T. Eriksen, Radiochim. Acta 82, 287 (1998).

${ }^{4}$ T. Eriksen, M. Jansson, and M. Molera, Eng. Geol. (Amsterdam) 54, 231 (1999).

${ }^{5}$ M. Molera and T. Eriksen, Radiochim. Acta 90, 753 (2004).

${ }^{6}$ S. Melchionna, S. Succi, and J.-P. Hansen, Int. J. Mod. Phys. C (to be published).

${ }^{7}$ D. Moroni, B. Rotenberg, J.-P. Hansen, S. Succi, and S. Melchionna, Phys. Rev. E (submitted).

${ }^{8}$ B. Baeyens and M. Bradbury, J. Contam. Hydrol. 27, 199 (1997).

${ }^{9}$ E. Tertre, G. Berger, S. Castet, M. Loubet, and E. Giffaut, Geochim. Cosmochim. Acta 69, 4937 (2005).

${ }^{10}$ M. Bradbury and B. Baeyens, Near Field Sorption Data Bases for Compacted MX-80 Bentonite for Performance Assessment of a High-Level Radioactive Waste Repository in Opalinus Clay Host Rock (Paul Scherrer Institut, Villigen, Switzerland, 2003).

${ }^{11}$ N. T. Skipper, G. Sposito, and F. C. Chang, Clays Clay Miner. 43, 294 (1995).

${ }^{12}$ F. C. Chang, N. T. Skipper, and G. Sposito, Langmuir 11, 2734 (1995).

${ }^{13}$ V. Marry, P. Turq, T. Cartailler, and D. Levesque, J. Chem. Phys. 117, 3454 (2002).

${ }^{14}$ N. Malikova, A. Cadène, V. Marry, E. Dubois, P. Turq, J.-M. Zanotti, and S. Longeville, Chem. Phys. 317, 226 (2005).

${ }^{15}$ N. Malikova, V. Marry, J.-F. Dufréche, C. Simon, P. Turq, and E. Giffaut, Mol. Phys. 102, 1965 (2004).

${ }^{16}$ N. Malikova, V. Marry, J.-F. Dufréche, and P. Turq, Curr. Opin. Colloid Interface Sci. 9, 124 (2004). 
${ }^{17}$ T. Ala-Nissila and S. C. Ying, Phys. Rev. Lett. 65, 879 (1990).

${ }^{18}$ S. C. Ying, Phys. Rev. B 41, 7068 (1990).

${ }^{19}$ G. Caratti, R. Ferrando, R. Spadacini, and G. E. Tommei, Physica A 246 15 (1997).

${ }^{20}$ G. Caratti, R. Ferrando, R. Spadacini, and G. E. Tommei, Phys. Rev. E 54, 4708 (1996).

${ }^{21}$ H. Risken, The Fokker-Planck Equation, 2nd ed. (Springer-Verlag, Berlin, 1989).

${ }^{22}$ L. Bocquet, Am. J. Phys. 65, 140 (1997).

${ }^{23}$ B. Bagchi, R. Zwanzig, and M. C. Marchetti, Phys. Rev. A 31, 892 (1985).

${ }^{24}$ M. Jardat, O. Bernard, P. Turq, and G. R. Kneller, J. Chem. Phys. 110, 7993 (1999).

${ }^{25}$ J.-F. Dufréche, M. Jardat, T. Olynyk, O. Bernard, and P. Turq, J. Chem. Phys. 117, 3804 (2002).
${ }^{26}$ J.-F. Dufréche, O. Bernard, S. Durand-Vidal, and P. Turq, J. Phys. Chem. B 109, 9873 (2005).

${ }^{27}$ GNUPLOT 4.0 URL: http://www.gnuplot.info

${ }^{28}$ B. Bagchi, J. Chem. Phys. 82, 5677 (1985).

${ }^{29}$ R. Zwanzig, Nonequilibrium Statistical Mechanics (Oxford University Press, New York, 2001).

${ }^{30}$ S. Lifson and J. L. Jackson, J. Chem. Phys. 36, 2410 (1962).

${ }^{31}$ G. Adam and M. Delbrueck, Reduction of Dimensionality in Biological Diffusion Processes, edited by A. Rich and N. Davidson (Freeman, San Francisco, 1968), p. 198.

${ }^{32}$ B. Rotenberg, A. Cadene, J.-F. Dufrêche, S. Durand-Vidal, J. C. Badot, and P. Turq, J. Phys. Chem. B 109, 15548 (2005).

${ }^{33}$ B. Rotenberg, J.-F. Dufrêche, and P. Turq, J. Chem. Phys. 123, 154902 (2005).

${ }^{34}$ N. Nandi and B. Bagchi, J. Phys. Chem. B 101, 10954 (1997). 